\title{
Preliminary Structural Design Using Topology Optimization with a Comparison of Results from Gradient and Genetic Algorithm Methods
}

\author{
Adam O. Burt ${ }^{1}$ and Michael L. Tinker ${ }^{2}$ \\ National Aeronautics and Space Administration, Marshall Space Flight Center, Alabama, 35812
}

In this paper, genetic algorithm based and gradient-based topology optimization is presented in application to a real hardware design problem. Preliminary design of a planetary lander mockup structure is accomplished using these methods that prove to provide major weight savings by addressing the structural efficiency during the design cycle. This paper presents two alternative formulations of the topology optimization problem. The first is the widely-used gradient-based implementation using commercially available algorithms. The second is formulated using genetic algorithms and internally developed capabilities. These two approaches are applied to a practical design problem for hardware that has been built, tested and proven to be functional. Both formulations converged on similar solutions and therefore were proven to be equally valid implementations of the process. This paper discusses both of these formulations at a high level.

\section{Nomenclature}

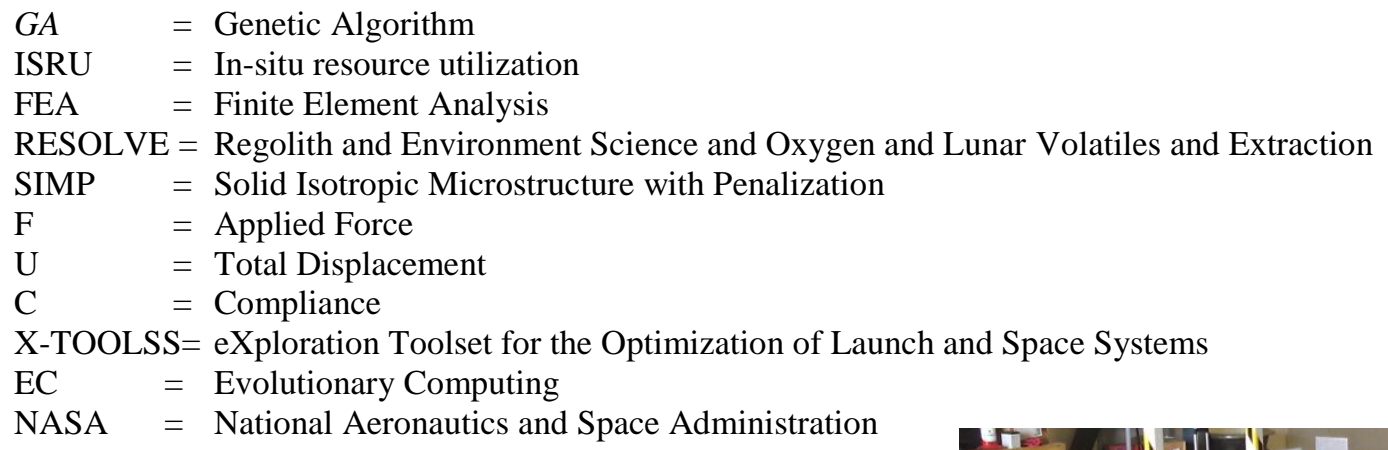

\section{Introduction}

$\mathrm{T}$ his paper presents the reinforcement design methodology used to design a mockup lander built for the Artemis Jr., Rover and RESOLVE demonstration in Hawaii. The lander mockup (Fig. 1) was designed to be capable of sustaining the weight of the rover while driving up the ramps and moving to a parking position on the lander. The final lander design consists of two decks for the placement of mock-up tanks and propulsion thrusters. The deck stiffeners were sized and formed using topology optimization techniques.

Topology optimization provides an excellent preliminary design tool for primary structures. It efficiently explores the design space by adding and removing material using Finite

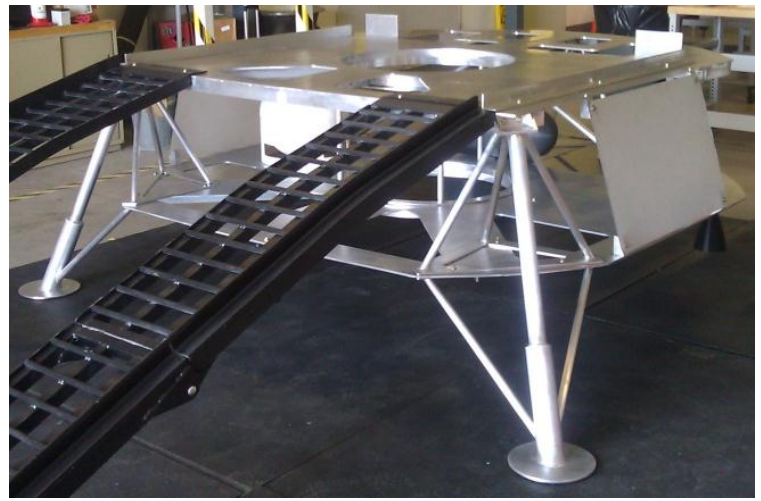

Figure 1: Manufactured Mockup Lander

${ }^{1}$ Aerospace Engineer, Thermal \& Mechanical Analysis Branch/ES22 Marshall Space Flight Center, AL 35812, AIAA Member.

${ }^{2}$ Assistant Division Manager; Systems Development, Integration \& Test/ES60; Marshall Space Flight Center, AL 35812, AIAA Associate Fellow. 
Element Analysis (FEA) to find the most efficient load paths. This process gives greater assurance that the final design, if the design space accurately reflects reality, will have acceptable margins of safety when the final stress analysis is performed. This allows more robust and efficient designs to be generated more quickly.

This paper is intended to objectively compare the two distinct approaches for topology optimization in terms of practical considerations. This paper is not intended to comment on or further the theory of topology optimization, but to present practical results as to the experience of its use in the design process. In the literature, great emphasis is placed upon utilizing the exact solution of the optimization. However, in practice, the exact optimum as presented by the results of the topology optimization is not always as useful due to manufacturing constraints, uncertainties in loads, and other uncertainties present in preliminary design. What is of importance is having a result that is close to the global optimal solution and then interpreting the results correctly to be incorporated into the design.

While not necessarily producing useful exact results, topology optimization can provide designers with a "map" of strategic material placement or reinforcement placement (such as angles or I-beams). Interpreting the shape of the resulting geometry can provide the designer with a starting point design that has higher structural efficiency and quicker design time compared to conventional approaches.

With this in mind, speed of problem setup, speed of the final solution, and confidence the solution has the global optima are the metrics of importance when comparing these two approaches. For this problem (like many other problems), the ultimate driver for the final design was to minimize the total mass.

The primary goal of this research was to evaluate GA and gradient-based design methods for use in future design cycles. While the mockup planetary lander was not intended for flight, the techniques identified and explored in its design are directly being used in efforts to develop a flight pallet lander. Going through this process provided insight into how to interpret the results of topology optimization and explore which methods are appropriate to use early in the design process to have high impact on the final design.

This paper will provide the reader with a brief and high-level description of the topology optimization problem. Several references are provided for both the genetic algorithm formulation as well as the traditional methods that provide a much more detailed look at the process.

\section{Process of Topology Optimization}

Topology optimization is the process of using FEA to identify the optimal material distribution that creates the most efficient load path. This is accomplished by first discretizing an initial design space into finite elements and then using a selection process to remove elements from that design space using an objective function. Figure 2 shows the initial discretized two-dimensional design space for a cantilevered beam type model. The two primary approaches other than genetic algorithms are the Evolutionary Structural Optimization Methods ${ }^{2}$ and the Solid Isotropic Microstructure with Penalization (SIMP) approach originally developed by Bendsoe. $^{12}$ These approaches both trim away unnecessary elements (though the objective function for these approaches is different) until only the minimum amount of structure or material needed remains. The objective function typically consists of minimizing compliance or strain energy, which results in a structure that has maximized stiffness and therefore higher structural efficiency. ${ }^{5}$

\section{Description of Approaches}

The RESOLVE mockup lander deck was designed using two topology optimization approaches. First, the SIMP method was used as provided by the commercial implementation of Altair's Optistruct ${ }^{4}$, then a generational genetic algorithm using blended crossover was used to drive the topology optimization using an approach developed by the NASA eXploration Toolset for the Optimization Of Launch and Space Systems (X-TOOLSS) team (Section III B.).

There is some debate in the literature as to which method is most appropriate ${ }^{8,10}$ therefore, both methods were used for this design problem and the results compared. These methods were used to find the optimum locations to reinforce the planetary lander top and bottom deck plates given an out-of-plane loading. These two methods, while generally used to minimize the same objective function, approach the problem differently. First a brief and highlevel description of the SIMP method is given. There is extensive treatment of this approach in the literature ${ }^{5,13}$. A 
more detailed look at the GA formulation is given in this paper as this was a capability developed internally and was of primary research interest to the authors.

\section{A. SIMP Method in Topology Optimization}

For this paper, Altair's Optistruct was used to perform the optimization. Optistruct uses the SIMP method, which formulates the design space as a continuous function. Each element's density is assigned as a continuous variable with a range from 0 to 1 , with 0 meaning none of that element's material is needed, 1 meaning the entire element is needed and intermediate values representing fictitious material. The density is typically given with a penalization parameter that drives the optimization to discrete values and avoids intermediate densities as much as possible. ${ }^{13}$ Then a gradient-based optimization algorithm solves the topology optimization problem using an iterative approach by evaluating the objective function, assessing the sensitivities of the design variables, and then choosing the next step (this information was obtained using Optistruct User's Guide to ensure that this was the approach implemented $)^{4}$.

The SIMP method for topology optimization has found widespread use in the literature and commercial implementations because of its quick convergence and its capability to handle very large design spaces, i.e., very dense meshes ${ }^{8}$. However, gradient-based algorithms have the disadvantage of potentially getting trapped in local optima, and do not inherently search the entire design domain ${ }^{10,11}$. This is where genetic algorithms have found roles in topology optimization. The following sections go into high level detail about how genetic algorithms work and how they are applied to the topology optimization problem.

\section{B. Genetic Algorithm-Based Topology Optimization}

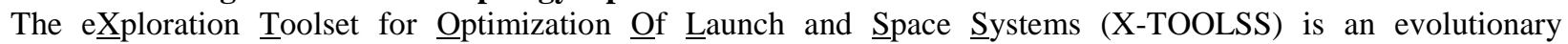
computation (EC) toolset that includes GAs and other types of ECs. X-TOOLSS is currently being developed by NASA Marshall Space Flight Center and university partners ${ }^{6,7,9}$. Genetic algorithms are based on the "survival of the fittest" concept. They develop optimal solutions for prospective designs by eliminating weak candidate designs. The power of EC techniques lies in their ability to discover unique, innovative, and often non-intuitive designs by thoroughly interrogating an entire design space. Furthermore, ECs drive the whole system to a global optimum and are able to avoid becoming "trapped" in local optima. The XTOOLSS GA capability was used to drive the topology optimization.

The GA topology optimization is formulated differently than the gradient-based approach. First, the setup of the design space is not based upon varying the density of each element. Instead, the topology problem is formulated as a void-solid problem where a set of discrete design variables corresponding to each element has only two values, 1 or 0 ("on" or "off") $)^{1,3}$. This is also known as a bit-array presentation $^{10}$. The GA use the bit-array as chromosomes to turn on or off specific elements, which adds and removes material to minimize the objective function (discussed in Section IV. B), finding the most efficient load path.

However, for this implementation, mesh connectivity becomes an issue. Solutions exist in the design space that do not have a continuous path connecting all elements to the boundary conditions of the finite element model. If these solutions occur they are highly penalized (compared to the objective function) based on the number of "groups" of disconnected elements that remain. An example of an entirely disconnected topology is shown in Figure 3. This helps to drive the algorithm back toward a connected solution. The other issue that occurs is that it is possible to have one group of elements connected to the boundary conditions with many other groups of elements that are disconnected entirely (demonstrated in Figure 4). For these cases, the groups of disconnected elements are simply switched to void leaving only the connected group remaining ${ }^{1}$ (result demonstrated in Figure 5). However, as stated by Wang, S. Y., et. al, ${ }^{10}$ this formulation has

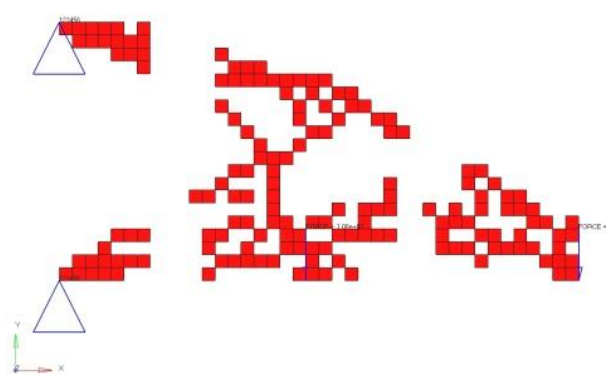

Figure 3: Disconnected Topology

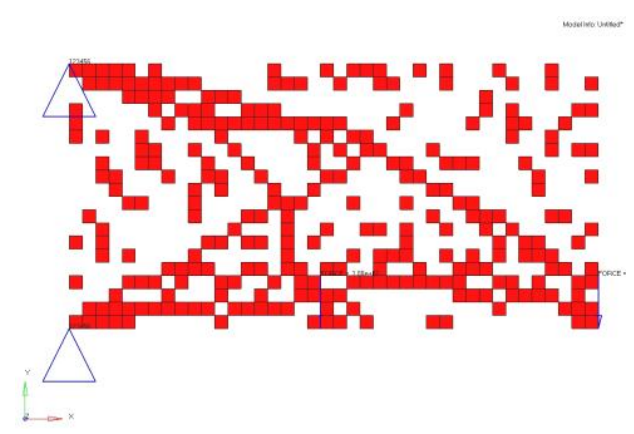

Figure 4: Groups of Disconnected Elements difficulty in providing the appropriate information back to the GA as many elements that are not used in the function evaluation remain in the design space and therefore incurs a higher computational cost. ${ }^{10}$ Therefore the time to convergence can be greatly increased. Future work will include efforts 
to alleviate this issue, but as shown in the results, it does not inherently invalidate the process; it does however, significantly decrease the efficiency of the optimization. Mesh connectivity is stated as being one of the major challenges for genetic algorithm-based topology optimization ${ }^{10}$.

Non-gradient based methods have been contested in the literature as being inefficient, as well as not providing significantly better results than traditional gradient-based methods $^{8}$. However, there are several potential advantages of GA-based optimization. First, as mentioned previously, using GAs can provide a global search of the design space, whereas methods such as the SIMP method can be trapped in local optima. Another advantage of GA's is their ability to handle non-analytic functions, as no derivative information is required. This means the analytic setup of the problem can be simplified, at the cost of computational efficiency, but for a designer this

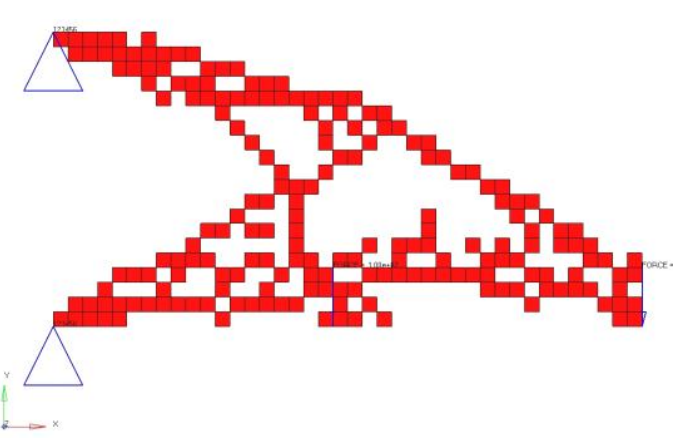

\section{Figure 5: Topology after Filtering for Disconnected Groups} could be an attribute if there is not a clear understanding of how the design space and structural responses are related and could help to provide insight into the problem.

There are, however, several disadvantages to using GAs for topology optimization. First, GA's require a very large number of function evaluations, on the order of tens of thousands, and this number is also directly related to the number of design variables. The problem is formulated as a void-solid problem and the size of the design space can be calculated as $2^{\text {Number of Elements }}$. This requires significant function evaluations, thus making this approach somewhat unattractive. However, if an exact result is not needed, only the implication of a global solution, and the results of the topology optimization problem are going to be interpreted and redesigned by a human designer (as in this case), then this problem can be overcome by reducing the design space (decreasing the mesh density) to provide an approximate look at the optimum material distribution.

By comparing the GA results to the gradient results it can give the designer confidence that an optimal solution has been found. The following sections will discuss the design space setup and results of the optimization for each method.

\section{Optimization Setup and Results}

The goal for this problem was to determine a reinforcement design that takes the entire load, leaving the top plate unloaded and as thin as possible to reduce mass. The formulation of this problem is slightly different from most topology optimization problems as it is an out-of-plane loading problem, where most problems are performed for inplane loading scenarios such as the cantilever beam model.

A Finite Element Model (FEM) of the top deck was created as shown in Figure 6. As this was the only design area of interest for this problem, the finite element model simulated the legs using conservative boundary conditions. Pinned boundary conditions were used to represent the lander legs. The inner ring was also pinned in locations where support struts were to be located attaching the top and bottom deck of the lander. In Optistruct only one load case can be used for the compliance minimization problem, therefore the primary load case of interest would be for the rover resting on the top deck. This was represented by applying $1 / 4$ of the rover weight at each of the four expected wheel locations.

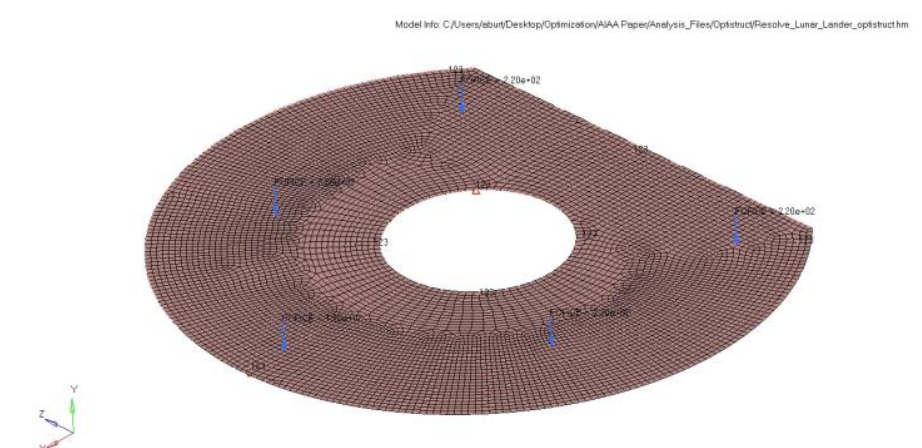

Figure 6: Optistruct Design Space for Resolve Lander Upper Deck Optimization 


\section{A. Optistruct Results for Lander Deck Design}

The gradient-based topology optimization was performed using Altair's Optistruct. The total design space can be seen in Figure 6. The deck was discretized into 7477 elements. For this case, every element was specified as a design variable. The topology optimization design problem is accomplished using compliance minimization. Compliance is defined as:

$$
C=F^{T} U
$$
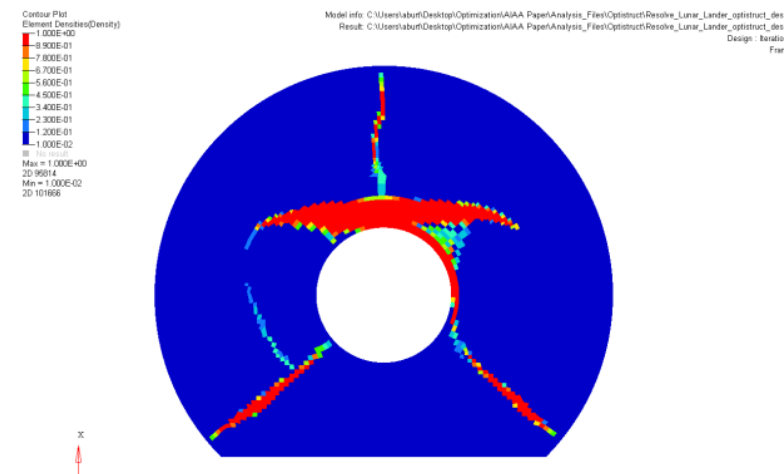

Figure 7: Gradient-Based Topology Optimization Results for Lander Deck

Where $\mathrm{F}$ is the force and $\mathrm{U}$ is the total displacement ${ }^{12}$. Typically the objective is to minimize compliance while constraining mass to be some fraction of the original. This acts to maximize the stiffness and thus the structural efficiency. This strategy was utilized for this optimization. The mass fraction constraint used was $10 \%$ over the unaltered design space mass. The von Mises stress design constraint was placed at $12 \mathrm{ksi}$ to avoid exceeding the allowable strength limits (this was factor of safety adjusted). This resulted in a feasible optimization solution that had acceptable margins of predicted strength and displacement. In Figure 7 for the topology optimization results, the contours show an element density of 1.0 (red) for areas where material is absolutely needed. Contours showing element density of 0.0 (blue) indicate void regions. As can be seen from

Figure 7, more material is needed in the front of the lander deck structure, but only straight stiffeners are needed to support the back of the deck. The regions of "needed" material constitute a map for how to reinforce the deck to reduce the minimum plate thickness to carry the load.

\section{B. Genetic Algorithm Optimization Results for Lander Deck Design}

In the genetic algorithm method, each solution in a design population is ranked based on a fitness (objective) function. For this case, the sum of the total mass of the system and total compliance of the system is used as the fitness.

$$
F I T=C+\text { Mass }
$$

Therefore, X-TOOLSS seeks to minimize the mass and compliance of the total system simultaneously, instead of constraining the mass fraction and minimizing compliance, as was done using Optistruct. Because of the afore-mentioned issues of computational efficiency, the design space was truncated to 1184 elements by reducing the mesh density to help increase convergence
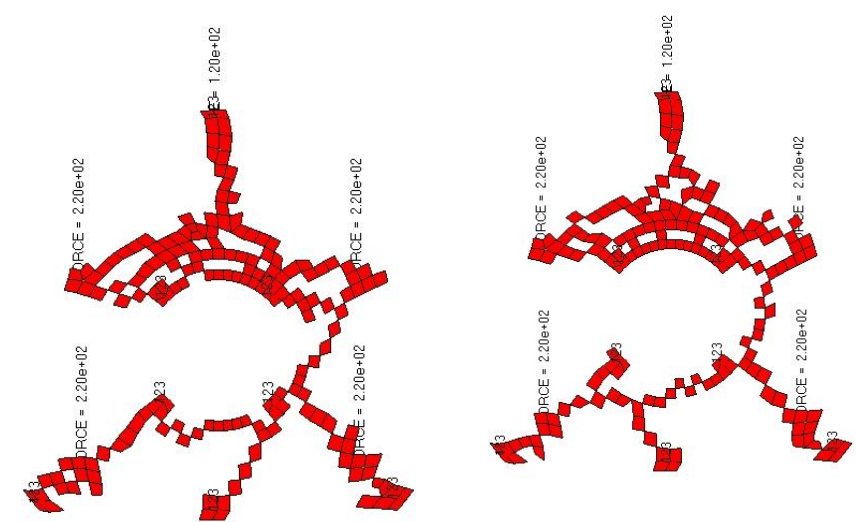

Figure 8: GA Optimization Results for Runs 1 and 2 speed. Genetic algorithms are non-deterministic, so it is important to perform several runs to get confidence in the result. These optimizations were run in parallel to decrease the total computational time. Figure 8 shows final results of two GA topology optimization runs. Both runs converged to similar solutions giving adequate confidence that an optimum has been found and that neither run was subject to premature convergence. 


\section{Comparison of Results}

\section{A. Comparison of Algorithm Results}

As can be seen by comparing Figures 7 and 8, both algorithms converged to very similar solutions. Because two different algorithms, totaling three runs, converged on the same solution, this design gives confidence that a good solution has been found.

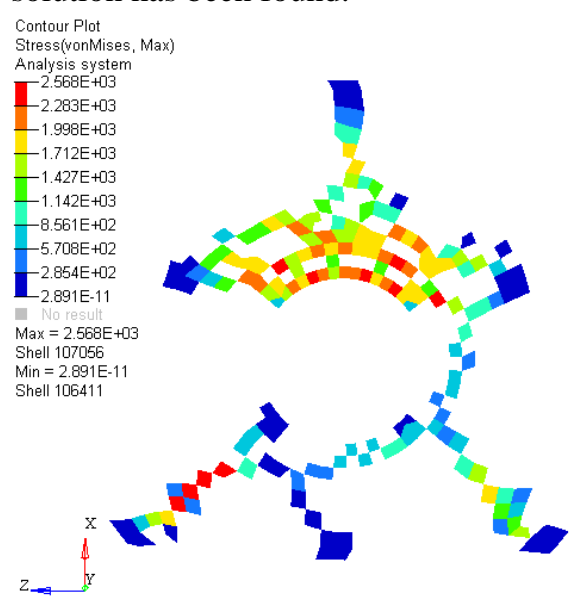

Figure 9: Stress Results for GA

Several conclusions could be drawn about each of these algorithms. First, it was evident from this experience that gradients are indeed a much faster implementation of the topology optimization problem, as the solution was able to converge in 26 iterations. However, several iterations of runs were needed to tweak the mass fraction constraint to find the lowest mass feasible design, resulting in a "manual" portion to the optimization. This was not necessary for the GA implementation, as both objectives were minimized simultaneously. As expected, the GA required a greater number of function evaluations (on the order of 26000) for both runs. Default settings in X-TOOLSS for mutation and crossover were used with only minor tuning to adjust the population size appropriately to maintain population diversity.

Comparing Figs. 7 and 8, it appears that the GA has determined that an extra member is needed in the negative $\mathrm{x}$-direction as compared with Optistruct. This is because that GA must maintain a topology that has all loads and boundary conditions connected by material, which is required for the void-solid formulation to be solved using Finite Element Analysis. This same constraint was not imposed by Optistruct. Because all elements exist and only density varies using the SIMP method, the boundary conditions can have very low density elements at the boundary conditions. To compare back to the GA results, the stress results show that the middle member identified is in fact unloaded. This can be seen in Figure 9. Therefore if this bottom-middle member is removed, the GA and gradient-based results are very similar.

\section{B. Interpreted Optimization Results}

As can be seen in Section IV, both the GA and gradient-based algorithms converged to similar solutions. These results give a reasonable level of confidence that a global near-optimum design has been found using both of these methods. The exact results of the optimization were not implemented, but were interpreted as a map to reinforce the top deck. This allows the designer to use the results, but also take into account fabrication considerations. Determining a structurally efficient load path allowed the top deck to be as thin as possible to reduce mass. The results also gave insight into where plate material could be removed without any structural penalty after the design had matured further.

The final design for the deck (shown in Figure 10) differs slightly from the results of topology optimization to address design concerns not taken into account by the load case used for the topology optimization. However, the basic reinforcement design is derived from the results of this study.

The finite element analysis of the final design showed positive margins of safety with respect to the 12,000 pounds per square inch constraint. Figure 1 shows the RESOLVE Lander mock-up based on the

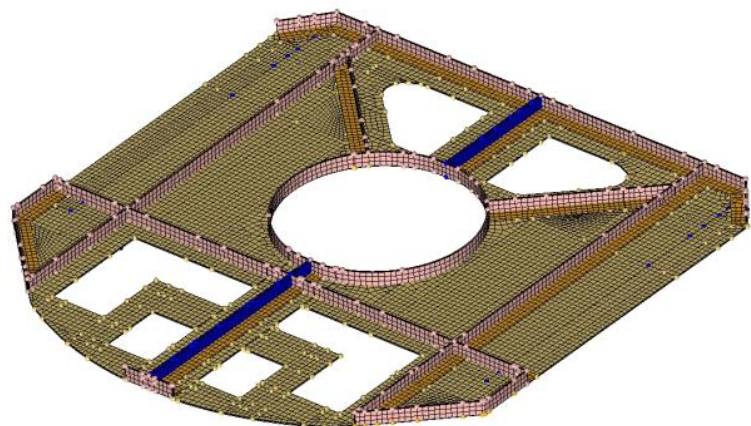
optimal design approaches described in this paper.

Figure 10: Final Design Finite Element Model 


\section{Conclusion}

Similar optimal designs for the RESOLVE mockup lander were obtained using GA-based and gradient-based topology optimization methods. Figures 7 and 8 show the results obtained using the two methods match well providing confidence an optimal reinforcement pattern has been found for the planetary lander deck structure. Topology optimization proved to be a useful tool to shorten the preliminary design cycle and still produce robust structures. The results also serve as a validation of the GA method for this design problem. By using GAs on what may be considered a simple problem, we have verified their use in the structural design cycle by comparing them with more traditional methods. In structural design cases other than topology optimization, GAs may prove to be useful by giving the designer more confidence a global optimum has been found. Because they can handle nonanalytic functions and require no derivative information, their setup is simplified, and they may be more attractive where time to set up the problem outweighs the function evaluation penalties inherent in non-deterministic approaches.

The results and lessons learned in this design process are being directly utilized in the design and development of a flight lunar pallet lander. Genetic Algorithms were proven to be valid when compared to more traditional methods, and it is hoped to continue using genetic algorithms for design challenges other than topology optimization.

\section{Acknowledgments}

A. O. Burt wishes to thank both Mr. Kevin Sykes and Mr. Doug Fox, P.E. Mr. Fox served as the analysis lead, and Mr. Sykes served as the lead design engineer for the RESOLVE mockup lander effort.

\section{References}

${ }^{1}$ Chapman, C. D., Saitou, K., and Jakiela, M. J., "Genetic Algorithms as an Apporach to Configuration for Topology Design," Journal of Mechanical Design, Vol. 116, No. 4, 1994, pp. 1005-1012.

${ }^{2}$ Huang, X., and Xie, Y.M., Evolutionary Topology Optimization of Contiuum Structures, John Wiley \& Sons, Ltd, Chichester, 2010.

${ }^{3}$ Jakiela, Mark J., Chapman, C., Duda, J., Adewuya, A., and Saitou, K., "Continuum Structural Topology Design with Genetic Algorithms," Computer Methods in Applied Mechanics and Engineering, Vol. 186, June, 2000, pp. 339-356.

${ }^{4}$ Optistruct, Software Package, Ver. 11.0, Altair Engineering Incorporated, 2013.

${ }^{5}$ Pererira, A., Talisch, C., Menezes, I. F. M., and Paulino, G. H,. "Checkerboard-free Topology Optimization using Polygonal Finite Elements," CILAMCE, 2010.

${ }^{6}$ SanSoucie, M. P., Hull, P.V., Irwin, R.W., Tinker, M. L., and Patton, B. W., "Trade Studies for a Manned High-Power Nuclear Electric Propulsion Vehicle," 1st Space Exploration Conference: Continuing the Voyage of Discove, Orlando, FL, AIAA, 2005.

${ }^{7}$ Schnell, A., et al., "Development of X-TOOLSS Preliminary Design of Space Systems Using Evolutionary Computation," 4th AIAA Multidisciplinary Design Optimization Specialist Conference, Schaumburg, IL, 2008.

${ }^{8}$ Sigmund, O., "On the Usefulness of Non-Gradient Approaches in Topology Optimization," Structural and Multidisciplinary Optimization, Vol. 43, No. 5, 2011, pp. 589-596.

${ }^{9}$ Tinker, M.L., et al, "Nuclear Electric Vehicle Optimization Toolset (NEVOT)," 10th AIAA/ISSMO Multidisciplinary Analysis and Optimization Conference, Albany, NY, 2004.

${ }^{10}$ Wang, S. Y., Tai, K., and Wang, M. Y., "An Enhanced Genetic Algorithm for Structural Topology Optimization," International Journal for Numerical Methods in Engineering, Vol. 65, No. 1, 2006, pp. 18-44.

${ }^{11} \mathrm{Wu}$, Chun-Yin, and Tseng, Ko-Ying, "Topology Optimization of Structures Using Modified Binary Differential Evolution." Structural and Multidisciplinary Optimization, Vol. 42, No. 6, 2010, pp. 939-953.

${ }^{12}$ Guest, J., Smith, L., "Topology Optimization of Continuum Structures Using HPM Encoded Genetic Algorithms," $49^{\text {th }}$ AIAA/ASME/ASCE/AHS/ASC Structures, Structural Dynamics, and Materials Conference, 2008.

${ }^{13}$ Rozvany, G. I. N., "Aims, Scope, Methods History and Unified Terminology of Computer-Aided Topology Optimization in Structural Mechanics,” Structural and Multidisciplinary Optimization, Vol. 21, No. 2, 2001, pp. 90-108 